\title{
Modeling PROFINET actions with timing pi-calculus
}

\author{
Wei $\operatorname{Jin}^{1 \mathrm{a}}$, Xin Gao ${ }^{1 \mathrm{~b}}$, Jing $\mathrm{Li}^{1 \mathrm{c}}$ \\ ${ }^{1}$ National Computer network Emergency Response technical Team/Coordination Center of China \\ (CNCERT/CC), \\ Beijing 100029, China

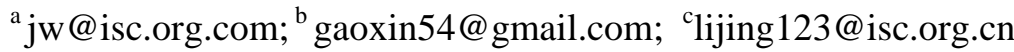

Keywords: PROFINET, pi-calculus, model

\begin{abstract}
PROFINET is a real-time protocol widely used in industrial control systems. To describe formally the semantics of PROFINET, we introduce the timing $\pi$-calculus, a new variant of the $\pi$ calculus, in this paper. The good feature of the timing $\pi$-calculus is that it can handle time elapse and timer events. We provide both its syntax and semantics. With the new calculus, we then model PROFINET, and give their precise semantics. Our formal framework may facilitate the reliability and consistency analysis of PROFINET.
\end{abstract}

\section{Introduction}

PROFINET[1][2] is defined by PROFIBUS and PROFINET International (PI) and is part of the IEC 61158[3] and IEC 61784[4] standards. It is developed for real time (RT) and isochronous real time (IRT) communication[5][6][7]. Nowadays, PROFINET is widely used in industrial control systems. But PROFINET is defined in natural language and suffers from inconsistency, ambiguity, and incompleteness. In the present paper we introduce a novel variant of the $\pi$-calculus [8][9] with timing abilities, which is called the timing $\pi$-calculus. The $\pi$-calculus is a process algebra proposed by Milner et: al in the 1980s with the goal of analyzing the behavior of mobile systems. Nowadays, the $\pi$-calculus has played an important role in modeling concurrent processes in computer science. But the typical $\pi$-calculus is not concerned about timing issues. Our timing $\pi$-calculus models time as distributed time units which is different from the Timer operator in a distributed $\pi$-calculus due to Berger and Yoshida [10]. The key point of the timing $\pi$-calculus is that actions are always atomic and can be finished in a flash. The elapse of time can only happen when no interactions can be done, that is, whenever there is an interaction that can happen, the system should never wait but finish the interaction.

So, with our new calculus, we can model PROFINET real-time actions and check some of their properties.

The rest of the paper is organized as follows. We introduce the syntax and semantics of the timing $\pi$-calculus in Section 2. Section 3 is devoted to modeling PROFINET actions with the timing $\pi$-calculus. We conclude the paper and present a direction for future work in Section 4.

\section{The Timing $\pi$-Calculus}

In this section, we introduce the timing $\pi$-calculus consisting of syntax and semantics, and recall the notion of bisimulation that relates two processes.

\subsection{Syntax}

Like traditional $\pi$-calculus, the simplest entities of the timing $\pi$-calculus are names. The only difference is that there is a special timing name $\varepsilon$ standing for a time unit. Here we call the traditional $\pi$-calculus names traditional names and range over them by lowercase letters $\mathrm{x}, \mathrm{y}, \mathrm{z}, \ldots$ Unlike the traditional names, $\varepsilon$ cannot be transferred within channels, and an occurrence of $\varepsilon$ means the elapse of one time unit. The prefixes of the timing $\pi$-calculus are displayed as follows:

$$
\pi::=\bar{x} y|x(y)| \varepsilon
$$


The first two prefixes mean sending and receiving a traditional name $\mathrm{y}$ via channel $\mathrm{x}$, respectively, and the third is a new timing prefix introduced here.

Definition 1: The syntax of the timing $\pi$-calculus processe is given by the following grammar:

$$
\begin{gathered}
P::=0|\pi . P| P+Q|P| Q|(v x) P| ! x(y) . P \\
\quad|[x=y] P| \operatorname{Timer}^{t}\langle x(y) . P ; Q\rangle
\end{gathered}
$$

Here, informally, these processes mean:

1) 0 is a process that does nothing.

2) $\bar{x} y . P$ sends $y$ through channel $x$ and then acts as process $P$.

3) $x(y) . P$ receives a traditional name, say $z$, through channel $x$ and then acts as process $P\{z / y\}$, where we are writing $P\{z / y\}$ for the substitution that maps $y$ to $z$ and is the identity for all other names in $P$.

4) c.P waits for a time unit and then acts as process $P$.

5) $P+Q$ stands for alternative selection. If one of the processes $P$ and $Q$ is performed, the other gets lost.

6) $P \mid Q$ stands for the parallel composition of processes $P$ and $Q$, which can either perform independently or interact via a shared channel.

$$
\begin{aligned}
& \text { Out } \overline{\bar{x} y \cdot P \stackrel{\bar{x} y}{\longrightarrow} P} \quad \text { OUT-DELAY } \overline{\bar{x} y \cdot P \stackrel{\epsilon}{\rightarrow} \bar{x} y \cdot P} \quad \text { INP } \overline{x(y) \cdot P \stackrel{x z}{\longrightarrow} P\{z / y\}} \\
& \text { INP-DELAY } \overline{x(y) \cdot P \stackrel{\epsilon}{\rightarrow} x(y) \cdot P} \\
& \text { SUM-L } \frac{P \stackrel{\alpha}{\rightarrow} P^{\prime}}{P+Q \stackrel{\alpha}{\rightarrow} P^{\prime}} \quad \text { SUM-DELAY } \quad \frac{P \stackrel{\epsilon}{\rightarrow} P^{\prime} \quad Q \stackrel{\epsilon}{\rightarrow} Q^{\prime}}{P+Q \stackrel{\epsilon}{\rightarrow} P^{\prime}+Q^{\prime}} \\
& \text { OPEN: } \frac{P \stackrel{\bar{x} y}{\longrightarrow} P^{\prime}}{(\nu y) P \stackrel{\bar{x}(y)}{\longrightarrow} P^{\prime}} \quad x \neq y \\
& \text { PAR-L } \frac{P \stackrel{\alpha}{\rightarrow} P^{\prime}}{P\left|Q \stackrel{\alpha}{\rightarrow} P^{\prime}\right| Q} \quad \text { bn }(\alpha) \cap f n(Q)=\phi \\
& \text { PAR-DELAY } \frac{P \stackrel{\epsilon}{\rightarrow} P^{\prime} \quad Q \stackrel{\epsilon}{\rightarrow} Q^{\prime}}{P\left|Q \stackrel{\epsilon}{\rightarrow} P^{\prime}\right| Q^{\prime}} \\
& \text { TIMER-DELAY1 } \overline{\text { Timer }^{t+1}\langle x(y) . P ; Q\rangle \stackrel{\epsilon}{\rightarrow} \operatorname{Timer}^{t}\langle x(y) \cdot P ; Q\rangle} \\
& \text { TIMER-DELAY2 } \frac{}{\operatorname{Timer}^{1}\langle x(y) \cdot P ; Q\rangle \stackrel{\epsilon}{\rightarrow} Q} \\
& \text { TIMER-TRIGGER } \frac{}{\text { Timer }^{t}\langle x(y) \cdot P ; Q\rangle \stackrel{x z}{\longrightarrow} P\{z / y\}} \\
& \text { PAR-TAU-L } \frac{P \stackrel{\tau}{\rightarrow} P^{\prime} \quad Q \stackrel{\epsilon}{\rightarrow} Q^{\prime}}{P\left|Q \stackrel{\tau}{\rightarrow} P^{\prime}\right| Q} \\
& \text { CLOSE-L } \frac{P \stackrel{\bar{x}(y)}{\longrightarrow} P^{\prime} \quad Q \stackrel{x y}{\longrightarrow} Q^{\prime}}{P \mid Q \stackrel{\tau}{\rightarrow}(\nu y)\left(P^{\prime} \mid Q^{\prime}\right)} \quad y \notin \mathrm{fn}(Q) \quad \text { COM-L } \quad \frac{P \stackrel{\bar{x} y}{\longrightarrow} P^{\prime} \quad Q \stackrel{x z}{\longrightarrow} Q^{\prime}}{P\left|Q \stackrel{\tau}{\rightarrow} P^{\prime}\right| Q^{\prime}\{y / z\}} \\
& \text { RES } \frac{P \stackrel{\delta}{\rightarrow} P^{\prime}}{(\nu y) P \stackrel{\delta}{\rightarrow}(\nu y) P^{\prime}} \quad y \notin n(\delta) \quad \text { CONG: } \quad \frac{P^{\prime} \equiv P \stackrel{\delta}{\rightarrow} Q \equiv Q^{\prime}}{P^{\prime} \stackrel{\delta}{\rightarrow} Q^{\prime}} \\
& \text { REP-ACT } \frac{x(y) \cdot P \stackrel{x z}{\longrightarrow} P^{\prime}}{! x(y) \cdot P \stackrel{x z}{\longrightarrow} P^{\prime} \mid ! x(y) \cdot P} \quad \text { REP-DELAY } \stackrel{ }{! x(y) \cdot P \stackrel{\epsilon}{\rightarrow} ! x(y) \cdot P}
\end{aligned}
$$

7) $(v x) P$ means the scope of name $x$ is restricted to $P$. 
8) ! $x(y) . P$ can be considered as an infinite composition $x(y) \cdot P|x(y) \cdot P| x(y) \cdot P \mid \ldots$.

9) $\operatorname{Timer}^{t}\langle x(y) . P ; Q\rangle$ is a timer process that waits through channel $x$ for $t$ time units, where $t$ is a strictly positive integer number. If a name $z$ is received within $t$ time units, it continues to act as $P\{z / y\}$; if nothing is received within $t$ time units, it changes to be $Q$.

Definition 2 (Binding): In each of $x(y) \cdot Q$ and ( $v y) Q$, the displayed occurrence of $y$ is binding with scope $P$. An occurrence of a name in a process is bound if it is, or it lies within the scope of, a binding occurrence of the name. An occurrence of a name in a process is free if it is not bound. We write $\mathrm{fn}(P)$ for free names in $P$, bn $(P)$ for bound names in $P$, and $n(P)$ for all names in $P$.

The concepts of Substitution and $\alpha$-conversion of traditional names are defined as the traditional $\pi$ calculus. Like the traditional $\pi$-calculus, the timing $\pi$-calculus processes that are $\alpha$-convertible are identified.

Definition 3 (Process context) A process context $C$ is, informally speaking, a process expression containing a hole, represented by [ ]. Formally, process contexts are given by the following syntax: $C::=[]|\pi \cdot C+Q|(v x) C|C| P|P| C \mid ! x(y) . C$

$\left|\operatorname{Timer}^{t}\langle x(y) . C ; Q\rangle\right| \operatorname{Timer}^{t}\langle x(y) . P ; C\rangle$

Definition 3 (Congruence) An equivalence relation $S$ on processes is a congruence if $(P, Q) \in S$ implies $(C[P], C[Q]) \in S$ for every context $C$.

Definition 4 (Structural congruence) Structural congruence, written $\equiv$, is the smallest congruence on processes that satisfies the following axioms:

1) item Changing bounded names ( $\alpha$-conversion)

2) $P+Q=Q+P$

3) $P|0 \equiv P, P| Q \equiv Q \mid P$, $P|(Q \mid R) \equiv(P \mid Q)| R$

4) $(v x) 0 \equiv 0,(v x)(v y) P \equiv(v y)(v x) P,(v x)(P \mid Q) \equiv P \mid(v x) Q$ if $x \notin f n(P)$

5) $! x(y) \cdot P \equiv x(y) \cdot P \mid ! x(y) \cdot P$

\subsection{Semantics}

Here we supply an account of action in the timing $\pi$-calculus by defining a family of labeled transition rules on processes. The transition relation is defined by a series of inference rules.

Definition 4 (Events): The transition rules are labeled by events which are given by the following grammar:

$$
\delta::=\bar{x} y|\bar{x}(y)| x y|\tau| \varepsilon
$$

We write Event for the set of events and range over them by $\delta, \eta, \ldots$ Besides, we call $\bar{x} y, \bar{x}(y)$, $x y$ and $\tau$ actions and range over them by $\alpha, \beta, \ldots$

Definition 5 (Transition Rules): The transition relation, $\{\stackrel{\delta}{\longrightarrow} \mid \delta \in E\}$, is defined in Table I.

\subsection{Bisimulation}

In process algebra theory, an important aspect is to find out when two processes act in the same way. There are many relations over processes that capture the behavioral equivalences of two processes. The notion of strong bisimulation seems to be the most natural one among them.

Definition 6 (Strong Bisimulation): A binary relation $S$ over processes is called a strong bisimulation if, whenever $(P, Q) \in S$,

1) if $P \stackrel{\delta}{\longrightarrow} P^{\prime}$, then there exists a process $Q^{\prime}$ such that $Q \stackrel{\delta}{\longrightarrow} Q^{\prime}$ and $\left(P^{\prime}, Q^{\prime}\right) \in S$.

2) if $Q \stackrel{\delta}{\longrightarrow} Q^{\prime}$, then there exists a process $P^{\prime}$ such that $P \stackrel{\delta}{\longrightarrow} P^{\prime}$ and $\left(P^{\prime}, Q^{\prime}\right) \in S$.

\section{Modeling PROFINET actions}




\subsection{PROFINET actions}

PROFINET can support three communication mechanisms: TCP/IP, real time (RT) and isochronous real-time (IRT). Intuitively, actions of PROFINET can be abstracted as follows:

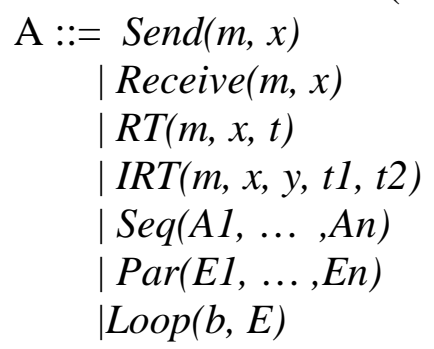

(TCP/IP Action)

(Receiving Action)

(RT Action)

(Sequence)

(IRT Action)

(Parallel)

(Loop)

Here, TCP, RT, and IRT are primary actions. Seq, Par and Loop are structured actions.

\subsection{Modeling PROFINET actions}

To give the semantics in terms of our timing $\pi$-calculus, a function ${ }^{[]_{\bar{y}}}$ is defined to map a PROFINET action to its timing $\pi$-calculus semantics (a timing $\pi$-calculus process). Here channel y indicates the accomplishment of the action and we assume that every PROFINET action has a unique y channel. For convenience, we write $\delta^{n}$ for a sequence of n events $\delta$, e.g., $\delta^{n}=\delta . \delta . \delta . \delta$. And if we are not interested with the parameter of an output, we just omit the parameter, e.g., we write $\mathrm{y}$ for $\mathrm{yx}$ if we do not care about the parameter $\mathrm{x}$. We write $\sum_{i \in I} P_{i}$ and $\prod_{i \in I} P_{i}$ for summation and parallel composition of processes $\left\{P_{i} \mid i \in I\right\}$, respectively. Furthermore, we abbreviate $\sum_{i \in I} \delta_{i} \cdot P$ as $\left(\sum_{i \in I} \delta_{i}\right) \cdot P$

\section{Primary Actions}

TCP/IP action: Sends a message by TCP/IP protocols.

$[T C P(m, x)]_{\bar{y}}=(v s)(\bar{m} s . \bar{s} x \cdot \bar{y})$

Here $m$ is the invoke channel of the message and $x$ is the message parameter been sent. We assume that every message has a unique name. The process firstly sends a restricted session channel $s$ through channel $m$, then sends the message through channel $s$. After that, a message is sent through channel $y$ indicating the accomplishment of the TCP action.

Receiving action: Receives a message.

$[\operatorname{Receive}(m, x)]_{\bar{y}}=m(s) \cdot s(x) \cdot \bar{y}$

Here $m$ is the receiving channel of the message and $x$ is the message parameter been received. The process waits for a session $s$ from channel $m$, then it receives message $x$ through $s$ and sends the accomplishment message through $y$.

RT action: Sends a message by the real-time protocol, if the message haven't been received in pre-defined time limit, return an error.

$[R T(m, x, t)]_{\bar{y}}=(v s)(v o)\left(\bar{m} s . \bar{s} x \cdot \bar{o} \mid \operatorname{Timer}^{t}\langle o(i) \cdot \bar{y} ; \bar{e}\rangle\right)$

Here $m$ is the invoke channel of the message and $x$ is the message parameter been sent. The timer in the process counts the elapse of time since the process has been activated, if no message through channel $o$, which indicates the accomplishment of sending message $x$, is received within $t$ time units, an exception will be thrown through predefined exception channel $e$.

IRT aciton: Sends a message by the isochronous real-time protocol. The message has to be sent between $t_{1}$ to $t_{2}$ time units. 


$$
\begin{aligned}
& {\left[\operatorname{IRT}\left(m, x_{1}, x_{2}, t_{1}, t_{2}\right)\right]_{\bar{y}}=(v s)(v o)(v u)\left(\bar{m} s \cdot \bar{s} x_{1} \cdot \bar{o}\right.} \\
& \left.\left|\operatorname{Timer}^{t_{1}}\left\langle o(i) \cdot \overline{y^{\prime}} ; \bar{e}\right\rangle\right| \text { Timer }^{t_{2}}\left\langle y^{\prime}(j) \cdot \bar{m} u \cdot \bar{u} x_{2} \cdot \bar{y} ; \bar{e}\right\rangle\right)
\end{aligned}
$$

Here $m$ is the invoke channel of the message and $x_{1}$ is the real-time message parameter and has to be sent in $t_{1}$ time unites, $x_{2}$ is the common message, it will be sent after the real-time message within the following $t_{2}$ time unites.

\section{Structured Actions}

Sequence: Sequence occurrence of inner actions $A_{1}, \ldots, A_{n}$. $A_{j+1}$ can be happened only after the happening of action $A_{j}$ has been finished.

$$
\left[\operatorname{Seq}\left(A_{1}, \ldots, A_{n}\right)\right]_{\bar{y}}=\left(v y_{1}\right) \ldots\left(v y_{n}\right)\left(\left[A_{1}\right]_{\bar{y}_{1}} \mid \prod_{j \in[1, n-1]} y_{j}(i) \cdot\left[A_{j+1}\right]_{\overline{y_{j+1}}}\right) \mid y_{n}(i) \cdot \bar{y}
$$

Here process $y_{j}(i) \cdot\left[A_{j+1}\right]_{\overline{y_{j+1}}}$ waits for the accomplishment of $A_{j}$ and process $y_{n}(i) \cdot \bar{y}$ waits for the accomplishment of $A_{n}$. After receiving a message through channel $y_{n}$, the accomplishment message of the $S e q$ action will be sent.

Parallel: Parallel occurrence of actions $A_{1}, \ldots, A_{n}$. Actions $A_{1}, \ldots, A_{n}$ can be happened simultaneously.

$$
\left[\operatorname{Par}\left(A_{1}, \ldots A_{n}\right)\right]_{\bar{y}}=\left(v y_{1}\right) \ldots\left(v y_{n}\right)\left(\prod_{j \in[1, n]}\left[A_{j}\right]_{\overline{y_{j}}} \mid\left(\sum_{j \in[i, n]} y_{j}(i)\right)^{n} \cdot \bar{y}\right)
$$

Here process $\left(\sum_{j \in[i, n]} y_{j}(i)\right)^{n} \cdot \bar{y}$ waits for the accomplishment of all inner actions.The accomplishment message of the Par action will be sent after all $A_{j}$ have been finished.

Loop: Loop of the inner action $A$. Action $A$ can be happened repeatedly.

$$
\begin{aligned}
& {[\operatorname{Loop}(b, A)]_{\bar{y}}=\left(v y^{\prime}\right)\left(\left([b=\text { false }] \bar{y}+[b=\text { true }] \overline{y^{\prime}}\right)\right.} \\
& \left.\mid ! y^{\prime}(i) .\left([b=\text { true }][A]_{\bar{y}^{\prime}}+[b=\text { false }] \bar{y}\right)\right)
\end{aligned}
$$

Here $b$ stands for a boolean expression. The process is started by the sub process $[b=$ false $] \bar{y}+[b=$ true $] \overline{y^{\prime}}$. If $b$ equals false, then $y$ will be chosen, and the action acts like an empty loop. Otherwise, the sub process $! y^{\prime}(i) .\left([b=\right.$ true $][A]_{y^{\prime}}+[b=$ false $\left.] \bar{y}\right)$ will be activated and starts to loop the inner action $A$.

\section{Conclusions}

The paper introduces a new variant of $\pi$-calculus called the timing $\pi$-calculus. The new $\pi$ calculus can handle timing events in communications. The modeling of PROFINET actions indicates the ability of the novel $\pi$-calculus, and the modeling itself gives a precise semantics for PROFINET actions.

In the future, we will formalize more PROFINET actions with our calculus and propose a behavioral typing system to check static properties of PROFINET actions.

\section{Acknowledgements}

Jing $\mathrm{Li}$ is the corresponding author of this paper. This work was supported by a grant from the National Key Technology R\&D Program (No. 2012BAH45B00).

\section{References}

[1] Profinet io part5: Application layer service definition, 2004.

[2] Profinet technology and application system description, 2006.

[3] Digital data communication for measurement and control - fieldbus for use in industrial control systems type 10: Profinet specification, 2003.

[4] Digital data communications for measurement and control part 1: Profile sets for continuous 
and discrete manufacturing relative to fieldbus used in industrial control systems, 2003.

[5] Robertini. A. Profinet: the future of the ethernet-based on automation. IPLnet Workshop, 2003

[6] Popp. The rapid way to profinet. PNO, pages 68-79, 2005.

[7] Specification profinet io version 2.0, 2005.

[8] Robin Milner. Communicating and Mobile Systems: the $\pi$-Calculus. Cambridge University Press, 1999.

[9] Davide Sangiorgi and David Walker. The $\pi$-Calculus: a Theory of Mobile Processes. Cambridge University Press, 2001.

[10] Martin Berger and Nobuko Yoshida. Timed, distributed, probabilistic, typed processes. Programming Languages and Systems, 4807:158-174, 2007. 